\title{
The Pharmacological Study of Zingerone.
}

\author{
$\mathrm{Br}$ \\ RISABURO DOI. \\ (土居利三郎) \\ (From the Pharmacological Laboratory of the Tohoku Imperial \\ University, Sendai.)
}

The rhizume of Zingiber officinal, Posc. or Radix Zingiberis has a peculiar aromatic odor and a pungent taste. It has been in use as a spice or to increase the appetite from the remotest times. Separation of the matter having this peculiar odor and taste has often been attempted, but without success. Pecently Dr Nomura set free this matter in its pure chemical form and has assigned it Zingerone. He further succeded in compounding the same substance synthetically. (The Transactions of the Chemical Society, 1917, Vol. III, p. 769.)

I have got from Dr Nomura the natural and artificial Zinger. ones and have made a pharmacological study of both of them. The results were the same in both cases. In the following accounts, therefore, I shall not make any distinction between the two.

Zingerone is a minute crystal, white in color. It is insoluable in water; but it can be easily dissolved in alcohol and olive oil. Hence, in experimentation its solution in olive oil or in Ringer's containing two percent of alcohol was used according to the place where it was applied. When I mention the quantity of Zingerone, however, it ought to be undertood as the quantity of that matter, not of its solution.

\section{Its Local Action.}

Zingerone is a volatile substance. Its vapor lightly irritates the mucous membrane of the nose. When the tip of the tingue was touched by this substance a burning pungency was felt. But it 
seemed that rabbit could stand it well. $1.0 \mathrm{grm}$. of Zingerone mixed with 100 grms. of the refuse of bean curd given to a rabbit was found to have been eaten up in a few hours. When one percent solution was applied to the mucous membrane of the mouth or the conjunctiva, only a slight congestion followed. The skin, when Zingerone was applied to it, showed no change.

\section{General Action.}

General Symptoms.

1) Frog.

When $0.5 \mathrm{mgrm}$. of Zingerone was injected into the abdominal lymph sac of Rana esculenta weighing about 20 grms. no change was seen. When 1.0 to 2.0 mgrms. was given, the respiration became a little slower and a slight paralysis followed. But soon it was restored to the normal state. When 3.0 to 5.0 mgrms was given, the respiration became slower and irregular and then ceased. The spontaneous and reflex movements became gradually weaker and finally disappeared. But there was no noticeable change in the beats of the heart. The animal continued to be in this state for a few minutes and gradually recovered from it. And after an hour or two, it was entirely restored to the normal state.

Experiment I. Rana esculenta, 20.0 grms.

2:20. 5.0 Zingerone is injected into the abdominal sac.

$2: 25$. It lies down. A slight dilation of the pupils. Slow respiration. No spontaneous movement. Slow reflex movements in response to mechanical stimuli.

2:40. Respiration slow and irregular. No reflex movement. Corneal reaction very weak.

$2: 45$. Respiratory movements stop. No corneal reaction. Beats of the heart still going on.

3:00. Respiration begins slowly and irregularly. Reflex movements are seen.

4:00. Perfect return to the normal state. Now and then leaping movements are seen.

In other experiments on the frog, it was found that even at the stage at which the animal was completely paralyzed by Zingrone, stimulation of the sciatic nerve caused a normal contraction of the gastrocnemius.

From the above experiments we may conclude that Zingerone paralyses the functions of the nervous system of the frog and so brings about a loss of motion. The appearance of and recovery from the effect is very quick. From the fact that the skin secretion and 
urine of the animal to which Zingerone is given have no odor peculiar to it, it is to be inferred that this matter is soon decomposed in the body and loses its special action.

2) Rabbit.

When 1.0 grm. of Zingerone per kilo body weight was given stomachally to a rabbit no noticeable change was seen. When the same quantity in olive oil solution was injected into the subcutaneous tissues the respiration became quicker and the blood vessels of the ear dilated in a slight degree. But all these symptoms disappear in an hour or two.

When 10,0 mgrms of Zingerone was injected into the ear vein of a rabbit the quick respiration of transitory character followed. The same phenomenon was to be seen in an animal which was anaesthetized with urethane. When 0.1 grm. was given to a rabbit the respiration became quicker for a time, but soon got slower and showed a sign of narcosis. After a few minutes the respiration entirely ceased. The spontaneous and reflex movements also disappeared. But at this time, when artificial respiration was applied, the respiratory movement was restored in a few minutes. The spontaneous and reflex movements gradually resumed their normal activities.

Experiment II. Rabbit, weighing 1.9 kilos.

10:00. The injection of $1 \%$ Zingerone solution into the ear vein begins. The respiration becomes quicker.

10:03. Respiration very slow. Relaxation of muscles. Contraction of the pupils.

10:04. The injection ends. (The quantity injected being about 20 c.c.) Standstill of respiration. No movement. Loss of the corneal reaction. Beats of the heart still going on. Immediately artificial respiration is instituted.

$10: 06$. Irregular respiration begins. Corneal reaction is seen.

$10: 10$. The ordinary attitude of the body is taken. The respiration becomes normal. The pupils dilatate to the former size. A sign of slight fatigue.

12:00. Normal, it takes food.

From this experiment, we see that Zingerone paralyses the central nervous system of the rabbit and canses a total loss of motion just as in the case of the frog.

That the preliminary quickning of the respiration is an effect of Zingerone and not due to alcohol contained in the solution could be proved by the control experiment. 'Transitoriness of effect may be due to the fact that Zingerone is easily decomposed in the body. The fact that the same symptom is not seen when applied into the 
stomach is accounted for by its decomposition in the digestive organ or the comparative slowness of its absorption by the latter.

Motor nerve ends and skeletal muscle. We have already seen that Zingerone causes the paralysis of voluntary and reflex movements of a frog by its action on the central nervous system. But we can not exclude that a slight paralysis of peripheral organs participates in it. To decide this question the following experiment was performed.

A frog, one of its hind legs being ligatured, was given a sufficient quantity of Zingerone. When the animal became complete motionless, the exitability of the sciatic nerve and the gastrocnemius was examined by electric stimulation. No difference, however, was found between the both sides. We may therefore conclude that Zingerone causes a paralysis of movements by its action on the central nerrous system only and not on the peripheral organs.

This substance, however, is not absolutely indifferent for the muscle, for isolated gastrocnemius of a frog, when it is immersed in a strong solution, such as 0.01 per cent, loses its exitability.

Smooth muscle. The abdominal wall of a rabbit anaesthetized by urethane was cut open and the stomach and the intestine were exposed. A catheter was inserted into the stomach or the small intestine and zingerone solution was injected into it.

When it was introduced, that part which was touched by the solution relaxed and its peristalsis became slower. The blood vessels dilated. When the solution was injected into a vein the relaxation of the intestine and the final cessation of its peristalsis followed. But this was only for a while.

A piece of the small intestine taken from a rabbit was suspended in a oxygenated Ringer's solution kept at the body temperature. The movements of the intestine were recorded by means of a lever on a revolving paper.

In $0.002 \%$ of Zingerone solution, the intestine relaxed a little and its peristalsis became weaker. But when the concentration was increased to $0.01 \%$ the noticeable relaxation and the final cessation of peristalsis followed. But gradually tone and peristalsis were resumed. At this time the further application of the solution would repeat the same phenomenon.

When the movements ceased a large quantity of pilocarpine applied to it had no effect. Electric stimulus had also no reaction. 
From these experiments, it appears that Zingerone paralyses the smooth muscle. Transitoriness of its action is probably due to its easy decomposition.

Circulation. The Heart. The chest of a frog was cut open and the heart was exposed. When $0.3 \mathrm{mgrm}$. of Zingerone was introduced under the skin, only a slight slowing of the beats followed. An isolated frog's heart nourished by artificial circulation showed no noticeable change by the administration of $0.3 \mathrm{mrgm}$. of Zingerone. When the quantity was increased to $0.5 \mathrm{mrgm}$. the rate and amplitude of the beats diminished steadily, until the heart stopped in a diastolic position. When at this stage the solution was interchanged with the fresh Ringer's solution, the heart began to beat and finally resumed the normal state. At the time of the standstill the ventricle reacted with one pulsation to a mechanical stimulus. But after the lapse of a little time, no reaction would occur. When it commenced to come to a standstill no atropine could prevent it. Hence we infer that Zingerone brings about a standstill of the heart not by stimulating the inhibitory but by paralyzing the motor apparatus of the heart. And that the heart muscles are affected is beyond doubt. But it is not clear whether the automatic centers are affected or not.

The Blood Vessels. The hind limbs of a frog were perfused with the Ringer's solution according to the Trendelenburg's method. If some Zingerone was introduced into the inflow tube the outflow of the liquid increased. This is the proof that Zingerone acts upon the walls of the blood vessels and causes their dilatation.

A rabbit was anaesthetized with urethane. The carotid blood pressure was recorded by a mercury manometer and Zingerone was injected into the vein. $0.01 \mathrm{grm}$. per kilogram of Zingerone produced no noticeable change. But when 0.1 gram. was given a remarkable change occured. The rate of heart beats considerably decreased and the blood pressure fall. But in that quantity, the animal usually recovered after, a while. Since the fall of the blood pressure was accompanied by the dilatation of the blood vessels of the ear, Zingerone acts upon the blood vessels and probably upon the heart as was seen in the experiment of the frog and causes the fall of blood preseure. 


\section{Summary.}

The subcutaneous injection of a large quantity of Zingerone to a frog causes motor paralysis of central origins.

When a large quantity of Zingerone is introduced into the alimentary canal of a rabbit, there is seen no other symptom than the relaxation of the intestines and the weakening of the peristalsis. When it is introduced into the blood vessel, the motor paralysis of central origin follows. The blood pressure may fall, but unless a large quantity is used the result is not noticeable. The above changes are temporary.

Radix Zingiberis as also its preparations is used as a spice or to increase the appetite. Zingerone has an aromatic odor peculiar to Radix Zingiberis. When infused into the blood it causes a motor paralysis. But when it is used internally, no symptom of intoxication is seen. The weakening of peristalsis may follow, but only when a large quantity is taken. Hence it may be used as a spice or to increase the appetite like other pungent stomachics. 